EPJ manuscript No.

(will be inserted by the editor)

\title{
Valence photoionization of small alkaline earth atoms endohedrally confined in $\mathrm{C}_{60}$
}

\author{
Mohammad H. Javani ${ }^{1}$, Meghan R. McCreary², Aakash B. Patel ${ }^{2}$, Mohamed E. Madjet ${ }^{3}$, Himadri S. Chakraborty², \\ and Steven T. Manson ${ }^{1}$ \\ 1 Department Physics and Astronomy, Georgia State University, Atlanta, Georgia 30303, USA \\ 2 Center for Innovation and Entrepreneurship, Department of Chemistry and Physics, Northwest Missouri State University, \\ Maryville, Missouri 64468, USA, e-mail: himadri@nwmissouri.edu \\ 3 Center for Free-Electron Laser Science, DESY, Notkestrasse 85, 22607 Hamburg, Germany
}

Received: date / Revised version: date

\begin{abstract}
A theoretical study of photoionization from the outermost orbitals of $\mathrm{Be}, \mathrm{Mg}$ and $\mathrm{Ca}$ atoms endohedrally confined in $\mathrm{C}_{60}$ is reported. The fullerene ion core, comprised of sixty $\mathrm{C}^{4+}$, is smudged into a continuous jellium charge distribution, while the delocalized cloud of carbon valence electrons plus the encaged atom are treated in the time-dependent local density approximation (TDLDA). Systematic evolution of the mixing of outer atomic level with states of the $\mathrm{C}_{60}$ valence band is found along the sequence. This is found to influence the plasmonic enhancement of atomic photoionization at low energies and the geometry-revealing confinement oscillations at high energies in significantly different ways: (a) The extent of enhancement is mainly determined by the strength of atomic ionization, giving the strongest enhancement for Be even though Ca suffers the largest mixing. But (b) strongest collateral oscillations are uncovered for $\mathrm{Ca}$, since, relative to $\mathrm{Be}$ and $\mathrm{Mg}$, the mixing causes the highest photoelectron production at confining boundaries of $\mathrm{Ca}$. The study paints the first comparative picture of the atomic valence photospectra for alkaline earth metallofullerenes in a dynamical many-electron framework.
\end{abstract}

PACS. 61.48.-c Structure of fullerenes and related hollow and planar molecular structures - 33.80.Eh Autoionization, photoionization, and photodetachment - 36.40.Cg Electronic and magnetic properties of clusters

\section{Introduction}

Laboratory techniques to entrap atoms and molecules in carbon fullerenes, forming endofullerenes, have seen recent improvements [1], increasing the feasibility of conducting spectroscopic experiments on endofullerenes in gas phases. In particular, measurements on photon induced ionization of these systems, as well as the empty fullerene but using the same technique, has become very visible over past years $[2-4]$. On the theoretical side, increasingly frequent studies on the topic of interactions of endofullerenes with external stimuli, namely, single photon $[5,6]$, multiphoton [7] and fast charged particle [8] fields have been reported, resulting in a steady growth of fundamental spectroscopic knowledge of atoms in a stable, material confinement, enriching the versatility of fullerene research. In addition, the spectroscopic information of endofullerenes is particularly valuable in the context of prospective technological applications of these systems in the fields from quantum computations [9] to photovoltaics [10] to the drug delivery [11].

Theoretical methods employed to obtain photoionization results for endofullerenes can be classified in four groups: (i) A simple semiclassical approach using the idea of a dynamical screening of the dielectric fullerene shell has produced a good qualitative description of the low energy photoemissions from the central atoms [12]. (ii) Important insights of energy dependent oscillations in atomic emissions at higher energies were obtained through calculations in which the confining $\mathrm{C}_{60}$ is modeled by static potentials, such as, zero-width delta-function potential [13] or finite-width square-well potential $[14,15]$. However, in either approach above, $\mathrm{C}_{60}$ electrons are completely omitted. Moreover, in the square well model, the width and depth of the potential can in principle be arbitrarily chosen as long as they together produce the measured $\mathrm{C}_{60}$ electron affinity, giving the potential a semi-empirical quality. (iii) Employing a one-center expansion type density functional scheme valence and core photoemissions of several guest atoms have also been performed [16]. While this approach is particularly superior in the single electron ground and continuum state description, by explicitly treating the truncated icosahedral structure of $\mathrm{C}_{60}$, the electronic collective motion was completely omitted. (iv) Finally, a method developed by our group that determines the ground state structure of delocalized elec- 
tron cloud, formed by the valence electrons of fullerene carbon atoms, moving in a jellium background of equivalent positive charge [17] in a local density approximation (LDA) framework. Photoionization results emanated from this LDA method, and from its time dependent (TDLDA) version at appropriate energies where many-electron effects dominate, have agreed well with experiments both in low energy plasmonic $[2,17]$ and high energy oscillatory [18] regions for free $\mathrm{C}_{60}$. Further, it has recently been shown that the oscillation signals in the Fourier reciprocal space of our single photoionization TDLDA results for $\mathrm{Xe} @ \mathrm{C}_{60}[19]$ in the $\mathrm{Xe} 4 \mathrm{~d}$ giant resonance range are qualitatively well reproduced [20] by the double photoionization measurements of $\mathrm{Xe}_{0} @ \mathrm{C}_{60}{ }^{+}$[4]. Particular other insights obtained from our TDLDA method include the prediction of (a) the oscillator strength transfer from the $\mathrm{C}_{60}$ plasmon to enhance the ionization of the confined atom $[21,22]$, (b) the existence of atom-fullerene hybrid levels of unusual spectral behavior [23], and (c) the significant reverse-modification of captor fullerene's own ionization signal by the encaged atom [24].

Using this TDLDA method we present herein the results of a comparative study of the photoionization of the first three alkaline earth metal atoms, $\mathrm{Be}, \mathrm{Mg}$ and $\mathrm{Ca}$, in $\mathrm{C}_{60}$. We consider the atoms located at the center of the $\mathrm{C}_{60}$ shell. In contrast to the case of alkali atoms, where the transfer of atomic outer electrons to the shell off-sets the atom's central position, in $\mathrm{Be}_{0} \mathrm{C}_{60}$ and $\mathrm{Mg} @ \mathrm{C}_{60}$ there is no electron transfer from the metal to the cage [25]. For $\mathrm{Ca}_{0} \mathrm{C}_{60}$ the situation is still unclear with the possibility of a partial transfer from Ca $4 \mathrm{~s}$ which may keep its atomic identity at least partly intact [25]. Therefore, we include $\mathrm{Ca}$ in our selection and explore, along the sequence, the evolution of the effect of the $\mathrm{C}_{60}$ plasmons as well as oscillatory energy-modulations on the captured atom's valence emission. The only previous study [16] was limited to the non-plasmonic effects and without the confining geometry based analysis of the oscillations.

\section{Description of the methodology}

\subsection{The ground state}

Density functional theory is used to describe the ground state electronic structure of the compounds using the same methodology employed earlier $[17,21]$. The jellium potential representing $60 \mathrm{C}^{4+}$ ions is constructed as a uniform charge density over a spherical shell with radius $R$ and thickness $\Delta$, augmented by a constant potential $V_{0}$ to insure quantitative accuracy [26]. $R$ is taken to be the known radius of $\mathrm{C}_{60}, 3.54 \AA$. The nucleus of the confined atom is placed at the center of the sphere. The Kohn-Sham equations for the system of a total of $240+N$ electrons $(N=4$ for Be, $N=12$ for $\mathrm{Mg}, N=20$ for $\mathrm{Ca}$, and 240 delocalized electrons from $\mathrm{C}_{60}$ ) are then solved to obtain the ground state wave function in the local density approximation (LDA), and the parameters $V_{0}$ and $\Delta$ are determined by requiring both charge neutrality and obtaining the experimental value, $7.54 \mathrm{eV}$, of the first $\mathrm{C}_{60}$ ionization

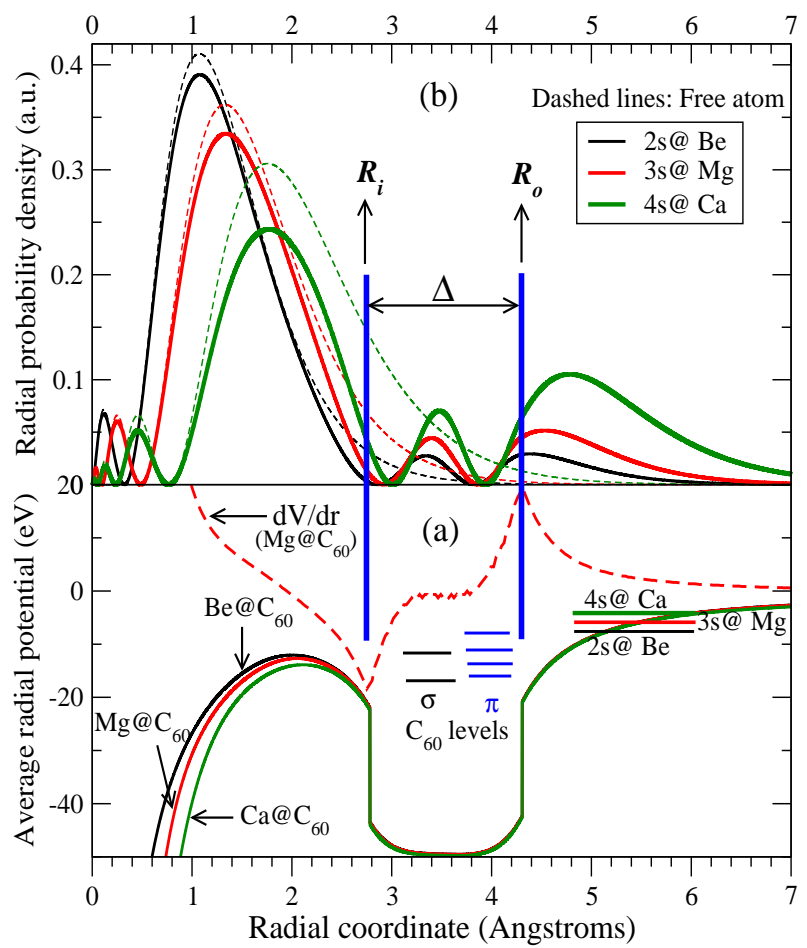

Fig. 1. (Color online) (a) Average radial LDA potentials for systems: $\mathrm{Be} @ \mathrm{C}_{60}, \mathrm{Mg} @ \mathrm{C}_{60}$, and $\mathrm{Ca} @ \mathrm{C}_{60}$. The radial derivative of the potential, a measure of the photoionizing force, is shown for the case of $\mathrm{Mg} @ \mathrm{C}_{60}$. The valence levels of the three confined atoms, and some high-lying $\pi$ and $\sigma$ levels of $\mathrm{C}_{60}$ are indicated. (b) Radial probability densities of free and confined atomic levels. The positions of inner and outer edges of the $\mathrm{C}_{60}$ hull are also indicated.

potential. This procedure yields a value of $\Delta$ of $1.5 \AA$, in excellent agreement with the value inferred from experiment [18].

In addition, we include an appropriate correction to eliminate unphysical electron self-interactions for the $i$-th subshell that render the LDA potential orbital-specific [6],

$$
\begin{aligned}
V_{\mathrm{LDA}}^{i}(\mathbf{r}) & =V_{\mathrm{jel}}(\mathbf{r})-\frac{Z_{\text {atom }}}{r}+\int d \mathbf{r}^{\prime} \frac{\rho\left(\mathbf{r}^{\prime}\right)-\rho_{i}\left(\mathbf{r}^{\prime}\right)}{\left|\mathbf{r}-\mathbf{r}^{\prime}\right|} \\
& +\left(V_{\mathrm{xc}}[\rho(\mathbf{r})]-V_{\mathrm{xc}}\left[\rho_{i}(\mathbf{r})\right]\right),
\end{aligned}
$$

where the terms on the right-hand side of the equation are, respectively, the jellium-electron, atomic nucleus-electron, electron-electron direct Coulomb and exchange-correlation potentials. As the exact form of $V_{\mathrm{xc}}$ is unknown in a local formalism like LDA (since the exact exchange interaction is non-local), we employ a widely used parametric formulation [27]. The successes and limitations of this jelliumbased description of $\mathrm{C}_{60}$ vis-á-vis experiments and quantum chemical calculations are discussed earlier [17], however no method can possibly beat the ease and ability of a jellium-approach to describe photoionization processes of nanometric finite systems by including important electron correlations.

Table 1 lists LDA single-electron configurations of $\mathrm{Be} @ \mathrm{C}_{60}$, $\mathrm{Mg} @ \mathrm{C}_{60}$ and $\mathrm{Ca} @ \mathrm{C}_{60}$ using Coulomb notations, and iden- 
Table 1. Ground state configurations of the endofullerene compounds.

\begin{tabular}{|c|c|c|}
\hline $\mathrm{Be} @ \mathrm{C}_{60}$ & $\mathrm{Mg} @ \mathrm{C}_{60}$ & $\mathrm{Ca} @ \mathrm{C}_{60}$ \\
\hline $\begin{array}{l}\text { 1s }(\text { Be } 1 \mathrm{~s}) \\
2 \mathrm{~s}\left(\mathrm{C}_{60} 1 \mathrm{~s}+\text { a weak atomic node) }\right. \\
3 \mathrm{~s}\left(\mathrm{C}_{60} 2 \mathrm{~s}+\text { a weak atomic node) }\right. \\
4 \mathrm{~s}(\mathrm{Be} 2 \mathrm{~s}+\text { two shell nodes })(2 \mathrm{~s} @ \mathrm{Be}) \\
\text { higher } \ell \text { states are } \mathrm{C}_{60} \text { states }\end{array}$ & $\begin{array}{l}1 \mathrm{~s}(\mathrm{Mg} 1 \mathrm{~s}) \\
2 \mathrm{~s}(\mathrm{Mg} 2 \mathrm{~s}) \\
2 \mathrm{p}(\mathrm{Mg} 2 \mathrm{p}) \\
3 \mathrm{~s}\left(\mathrm{C}_{60} 1 \mathrm{~s}+\text { two weak atomic nodes }\right) \\
3 \mathrm{p}\left(\mathrm{C}_{60} 2 \mathrm{p}+\text { one weak atomic node) }\right. \\
4 \mathrm{~s}\left(\mathrm{C}_{60} 2 \mathrm{~s}+\text { two weak atomic nodes }\right) \\
4 \mathrm{p}\left(\mathrm{C}_{60} 3 \mathrm{p}+\text { one weak atomic node) }\right. \\
5 \mathrm{~s}(\mathrm{Mg} 3 \mathrm{~s}+\text { two shell nodes) (3s@ } \mathrm{Mg}) \\
\text { higher } \ell \text { states are } \mathrm{C}_{60} \text { states }\end{array}$ & $\begin{array}{l}1 \mathrm{~s}(\mathrm{Ca} 1 \mathrm{~s}) \\
2 \mathrm{~s}(\mathrm{Ca} 2 \mathrm{~s}) \\
2 \mathrm{p}(\mathrm{Ca} 2 \mathrm{p}) \\
3 \mathrm{~s}(\mathrm{Ca} 3 \mathrm{~s}) \\
4 \mathrm{~s}\left(\mathrm{C}_{60} 1 \mathrm{~s}+\text { three weak atomic nodes) }\right. \\
3 \mathrm{p}\left(\mathrm{C}_{60} 2 \mathrm{p}+\text { two weak atomic nodes) }\right. \\
4 \mathrm{p}(\mathrm{Ca} 3 \mathrm{p}+\text { one weak shell node) } \\
5 \mathrm{~s}\left(\mathrm{C}_{60} 2 \mathrm{~s}+\text { three weak atomic nodes) }\right. \\
5 \mathrm{p}\left(\mathrm{C}_{60} 3 \mathrm{p}+\text { two weak atomic nodes) }\right. \\
6 \mathrm{~s} \text { (Ca } 4 \mathrm{~s}+\text { two shell nodes) (4s@ Ca) } \\
\text { higher } \ell \text { states are } \mathrm{C}_{60} \text { states }\end{array}$ \\
\hline
\end{tabular}

Table 2. Valence binding energies $(\mathrm{eV})$ of free and confined atoms.

\begin{tabular}{l|lll}
\hline & $\mathrm{Be} @ \mathrm{C}_{60}$ & $\mathrm{Mg} @ \mathrm{C}_{60}$ & $\mathrm{Ca} @ \mathrm{C}_{60}$ \\
\hline$\eta \mathrm{s}$ & -9.49 & -7.92 & -6.25 \\
$\eta \mathrm{s} @$ & -7.57 & -5.87 & -4.04 \\
\hline
\end{tabular}

tifies the nature of $\mathrm{s}$ and p orbitals. Several of these orbitals invoke additional radial nodes in order to satisfy the Pauli exclusion principle. However, with only exceptions of the atomic valence orbitals, effects of these extra nodes on the shape of other orbitals are extremely weak, virtually retaining these orbitals rather pure. The radial component of the LDA potentials, calculated by the occupancy weighted averaging of all the orbitals of an endofullerene, are presented in Fig. 1(a) for the three systems. As expected, the depth of the atomic cusp gradually increases as the size of the atom grows. But the potential in the $\mathrm{C}_{60}$ shell region remains unchanged. This is because, even though the orbital-specific potentials (not shown) corresponding to Pauli-modified atomic valence levels do alter over the shell region, the effect washes out in the averaging process, since these orbitals being s-type hold only two electrons.

Fig. 1(b) shows the radial probability densities of the valence electrons which are $2 \mathrm{~s}, 3 \mathrm{~s}$ and $4 \mathrm{~s}$, respectively, for the three free atoms. We use the symbol $\eta \mathrm{s} @$, with $\eta=2$, 3,4 , to denote the levels of the confined atom, while technically these wavefunctions are $4 \mathrm{~s}, 5 \mathrm{~s}$ and $6 \mathrm{~s}$ of the compound system due to the mixing induced by two additional nodes (Table. 1). As seen in Fig. 1(b), the relative strength of the mixing grows with the increase of the atomic size, consequences of which on the photoionization mechanism over the plasmon active energy region and beyond will be revealed in the following section. In Fig 1(a), the positions of the corresponding binding energies of captive atomic levels are included, the explicit values of which along with those for free atoms are noted in Table 2, that suggests the decrease of binding energies from the mixing. We also show in Fig. 1(a) a few high-lying $\pi$ (single-noded) and $\sigma$ (nodeless) $\mathrm{C}_{60}$ states in order to aid our later discussions.

\subsection{The photoionization treatment}

A time-dependent LDA (TDLDA) approach [17] is used to calculate the dynamical response of the compound to the external dipole field $z$. Since the molecule is rotationally invariant, the Green's function for a parameter $E$ can be expanded in spherical basis:

$$
G\left(\mathbf{r}, \mathbf{r}^{\prime} ; E\right)=\sum_{l m} G_{l m}\left(r, r^{\prime} ; E\right) Y_{l m}^{*}(\Omega) Y_{l m}\left(\Omega^{\prime}\right),
$$

where the radial component $G_{l m}\left(r, r^{\prime} ; E\right)$ satisfies the radial equation

$$
\begin{array}{r}
\left(\frac{1}{r^{2}} \frac{\partial}{\partial r} r^{2} \frac{\partial}{\partial r}-\frac{\ell(\ell+1)}{r^{2}}-V_{\mathrm{LDA}}+E\right) G_{l m}\left(r, r^{\prime} ; E\right) \\
=\frac{\delta\left(r-r^{\prime}\right)}{r^{2}}
\end{array}
$$

$G_{l m}$ is constructed with homogeneous solutions $j_{l}(r ; E)$ and $h_{l}(r ; E)$ of Eq. 3, satisfying boundary conditions at $r=0$ and $r=\infty$ respectively, as

$$
G_{l m}\left(r, r^{\prime} ; E\right)=\frac{j_{l}\left(r_{<} ; E\right) h_{l}\left(r_{>} ; E\right)}{W\left[j_{l}, h_{l}\right]},
$$

where the Wronskian

$$
W[j, h]=r^{2}[j(r) d h(r) / d r-d j(r) / d r h(r)]_{r=c}
$$

and is independent of the arbitrary constant $c$. Using the Green's function, the independent particle (IP) susceptibility is then constructed by the ground state singleelectron orbitals $\phi_{i}$ and energies $\epsilon_{i}$ as

$$
\begin{aligned}
\chi_{0}\left(\mathbf{r}, \mathbf{r}^{\prime} ; \omega\right) & =\sum_{i} \phi_{i}^{*}(\mathbf{r}) \phi_{i}\left(\mathbf{r}^{\prime}\right) G\left(\mathbf{r}, \mathbf{r}^{\prime} ; \epsilon_{i}+\hbar \omega\right) \\
& +\sum_{i} \phi_{i}(\mathbf{r}) \phi_{i}^{*}\left(\mathbf{r}^{\prime}\right) G^{*}\left(\mathbf{r}, \mathbf{r}^{\prime} ; \epsilon_{i}-\hbar \omega\right),
\end{aligned}
$$

where the index $i$ runs over the occupied states only.

The external perturbation $z$ representing the dipole interaction of electrons with the linearly polarized light, induces a frequency-dependent complex change in the electron density. This can, in principle, be written as

$$
\delta \rho(\mathbf{r} ; \omega)=\int \chi\left(\mathbf{r}, \mathbf{r}^{\prime} ; \omega\right) z^{\prime} d \mathbf{r}^{\prime}
$$


where the full susceptibility $\chi$ incorporates the dynamical electron correlation. Using instead the IP susceptibility (Eq. 6), the induced density can, equivalently, be written as

$$
\delta \rho(\mathbf{r} ; \omega)=\int \chi_{0}\left(\mathbf{r}, \mathbf{r}^{\prime} ; \omega\right) \delta V\left(\mathbf{r}^{\prime} ; \omega\right) d \mathbf{r}^{\prime}
$$

in which

$$
\begin{aligned}
\delta V\left(\mathbf{r}^{\prime} ; \omega\right) & =z+\delta V^{\prime}\left(\mathbf{r}^{\prime} ; \omega\right) \\
& =z+\int \frac{\delta \rho\left(\mathbf{r}^{\prime} ; \omega\right)}{\left|\mathbf{r}-\mathbf{r}^{\prime}\right|} d \mathbf{r}^{\prime}+\left[\frac{\partial V_{\mathbf{x c}}}{\partial \rho}\right]_{\rho=\rho_{0}} \delta \rho(\mathbf{r} ; \omega),
\end{aligned}
$$

where the second and third term on the right-hand side, collectively represented by $\delta V^{\prime}$, are, respectively, the induced change of the Coulomb and the exchange-correlation potentials. Obviously, besides including the external perturbation $z, \delta V$ incorporates the dynamical field $\delta V^{\prime}$ produced through many-electron interactions and, thereby, plays the role of an effective perturbation to the molecule.

The photoionization cross section is then finally evaluated in terms of $\delta V$ as the sum of independent subshell cross sections $\sigma_{n \ell \rightarrow k \ell^{\prime}}$, corresponding to a boundto-continuum dipole transition $n \ell \rightarrow k \ell^{\prime}$,

$$
\sigma_{\mathrm{PI}}(\omega)=\sum_{n \ell} \sigma_{n \ell \rightarrow k \ell^{\prime}} \sim \sum_{n \ell} 2(2 \ell+1)\left|\left\langle\phi_{k \ell^{\prime}}|\delta V| \phi_{n \ell}\right\rangle\right|^{2} .
$$

Obviously, replacing $\delta V$ by $z$ in Eq. (10), we find the independent particle (IP) LDA results of the absorption and ionization cross sections respectively.

\section{Results and discussions}

\subsection{Low energy plasmonic region}

Fig. 2 shows the TDLDA cross sections for the valence levels $\eta \mathrm{s} @$ of the three confined atoms, along with their free atomic counterparts, for photon energies up to $30 \mathrm{eV}$. (Mounted on the broad cross section shapes of the confined atoms are narrow single-electron autoionizing resonances which are beyond the scope of our discussions.) First, a confinement induced general trend of the Cooper minima, appearing at the above-threshold region, to shift higher in the energy is noted. Second, the energy range in Fig. 2 is also the region where the $\mathrm{C}_{60}$ giant plasmon is excited. Therefore, the results also show dramatic enhancements in the ionization of confined atoms, peaking at about $16 \mathrm{eV}$, which are expected from the atom- $\mathrm{C}_{60} \mathrm{dy}-$ namical multielectron coupling $[6,22,21]$. However, the degree of this enhancement is clearly different from one system to another; in particular 2s@ Be exhibits the largest cross section while $3 \mathrm{~s} @ \mathrm{Mg}$ the smallest.

In order to uncover the reason of this trend, consider the TDLDA dipole matrix element in Eq. 10, denoted by $\mathcal{M}_{\eta \mathrm{S} @}$, in a decomposed single and multielectron picture:

$$
\mathcal{M}_{\eta \S} @(E)=\mathcal{D}_{\eta \mathrm{s} @}(E)+\Delta \mathcal{D}(E)
$$

where $\mathcal{D}$ is the (LDA) matrix element involving the single electron operator $z$. The structure of $\Delta \mathcal{D}$, which obviously

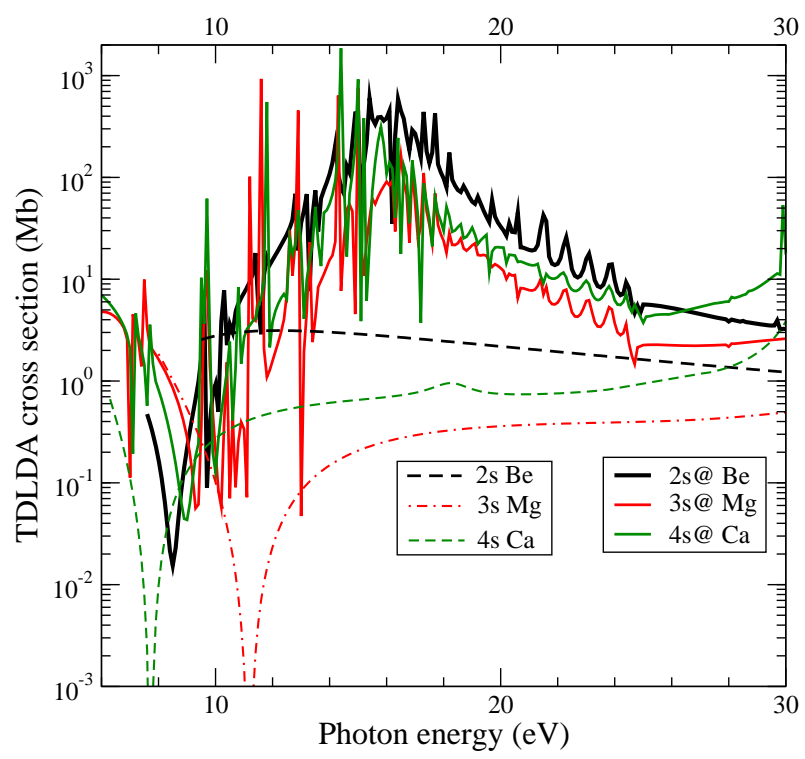

Fig. 2. (Color online) The valence ionization cross sections corresponding to confined and free atoms, for the three endofullerenes, calculated in TDLDA over the $\mathrm{C}_{60}$ plasmon region.

involves $\delta V^{\prime}$ [Eq. 9], can be alternatively expressed in the well known Fano scheme based on a perturbative interchannel coupling framework [21] as

$\Delta \mathcal{D}(E)=\sum_{n \ell} \int d E^{\prime} \frac{\left\langle\psi_{n \ell}\left(E^{\prime}\right)\left|\frac{1}{\left|\mathbf{r}_{\eta \mathrm{s} @}-\mathbf{r}_{n \ell}\right|}\right| \psi_{\eta \mathrm{s} @}(E)\right\rangle}{E-E^{\prime}} D_{n \ell}\left(E^{\prime}\right)$.

In Eq. 12, $\psi_{\eta \mathrm{s} @}(E)$ and $\psi_{n \ell}(E)$ are the unperturbed final continuum channel wave functions of $\eta \mathrm{s} @$ and $n \ell$ photoionization channels respectively, and the sum is over the photoionization channels of the atom plus all the delocalized electron (coherent) channels of the $\mathrm{C}_{60}$ shell. Separating out the atomic channels from the sum and associating those terms with the LDA matrix element $\mathcal{D}_{\eta \S} @$ we can rewrite Eq. 11 as

$$
\mathcal{M}_{\eta \S} @(E)=\mathcal{M}_{\eta \mathrm{s}}^{\text {atom }}(E)+\Delta \mathcal{D}_{\mathrm{C}_{60}}(E) .
$$

where the first term on the right hand side is approximately the TDLDA matrix element of the free atom and the second term, represented by Eq. 12 but without the atomic channels, embodies the plasmonic enhancement. The $\eta$ s@ photoionization cross section, $\sim\left|\mathcal{M}_{\eta \mathrm{s}} @\right|^{2}$, in TDLDA therefore involves the coherent superposition of the atomic TDLDA and the enhancement contributions:

$\sigma_{\eta \mathrm{s} @} \approx \sigma_{\eta \mathrm{s}}^{\text {atom }}+\left[\mathcal{M}_{\eta \mathrm{s}}^{\text {atom* }} \Delta \mathcal{D}_{\mathrm{C}_{60}}+\mathcal{M}_{\eta \mathrm{s}}^{\text {atom }} \Delta \mathcal{D}_{\mathrm{C}_{60}}^{*}\right]+\Delta \sigma_{\mathrm{C}_{60}}$

in which $\Delta \sigma_{\mathrm{C}_{60}} \sim\left|\Delta \mathcal{D}_{\mathrm{C}_{60}}\right|^{2}$.

The matrix element within the integral of Eq. 12 is known as the interchannel coupling matrix element. Going from $\mathrm{Be}$ to $\mathrm{Mg}$ to $\mathrm{Ca}$, the increasing mixing of $\eta \mathrm{s} @$ initial state wave function with $\mathrm{C}_{60}$ orbitals [Fig. 1(a)] ensures increasing overlaps, resulting in higher values of the interchannel coupling matrix elements. However, along the 
same atomic sequence there also arises a different effect that counters this trend as follows. Fig. 1(b) (and Table. 2) indicates a gradual decrease of the $\eta \mathrm{s} @$ binding energies from $\mathrm{Be}$ to $\mathrm{Ca}$, causing the valence level energetically moving away from the $\mathrm{C}_{60} \sigma$ and $\pi$ occupied bands [indicated in Fig. 1(b)]. Since the proximity of the ionization thresholds of interacting channels produces stronger overlaps between their continuum wavefunctions, the effect described above will lower the value of the coupling from Be to Ca. It is therefore expected that the competition of these two compensatory effects will keep the value of $\Delta \mathcal{D}_{\mathrm{C}_{60}}$, and hence $\Delta \sigma_{\mathrm{C}_{60}}$, largely unaltered. Consequently, the comparative differences in $\sigma_{\eta \S} @$ up the sequence will primarily be influenced by the free atomic matrix element $\mathcal{M}_{\eta \mathrm{s}}^{\text {atom }}$ via the interference term in the square bracket on the right hand side of Eq. 14. That is exactly what is found: The free atomic TDLDA matrix elements $\mathcal{M}$ must have increased from $\mathrm{Mg}$ to $\mathrm{Ca}$ to $\mathrm{Be}$, since their cross sections in Fig. 2 increase likewise. Therefore, from the above argument, this must also be the trend for the enhanced cross sections of the confined atoms, as clearly seen in the same figure.

\subsection{High energy oscillatory region}

At photon energies above the plasmonic range (roughly $>50 \mathrm{eV}$ ) the valence $\eta \mathrm{s} @$ ionization cross sections show oscillations as a function of the photon energy as seen in Figs. (3) for the three systems considered; the curves are seen to oscillate around the corresponding free atom $\eta \mathrm{s}$ cross sections. Since the many-electron effects largely weaken over this energy region, the single-electron LDA results, obtained by excluding the component $\delta V^{\prime}$ of the induced potential in Eq. 9, are enough to describe this oscillatory behavior. In Figs. 3(a) and (b) we also present results from Ref. [16] for $\mathrm{Be} @ \mathrm{C}_{60}$ and $\mathrm{Mg} @ \mathrm{C}_{60}$ that compare fairly well with the current LDA results within the limitation of their differences in formalism; for $\mathrm{Ca} @ \mathrm{C}_{60}$, Ref. [16] includes a multi-configuration splitting, making its comparison with our jellium-based result rather difficult.

Within the LDA framework the physical mechanism producing the oscillations can be qualitatively described by the acceleration gauge formalism. In this formalism, the dipole photoionization amplitude from Eq. 10, but ignoring correlations, can be expressed as

$$
\mathcal{D}_{\eta \mathrm{s} @} \sim\left\langle\phi_{k \mathrm{p}}\left|\frac{\partial V}{\partial r}\right| \phi_{\eta \mathrm{s} @}\right\rangle,
$$

which embodies the notion that the electron in the potential $V(r)$ requires a force $\partial V / \partial r$ to escape the system. This ionizing force offered by the average radial potential of the compound is displayed in Fig. 1(a), but only for $\mathrm{Mg} @ \mathrm{C}_{60}$, which produces characteristic peaks at the inner and the outer edges, $R_{i}(=2.79 \AA)$ and $R_{o}(=4.29$ $\AA$ ), of the $\mathrm{C}_{60}$ hull, suggesting the possibility of photoemissions from these locations. Note further in Fig. 1(a) that a rather strong force is also available in the central

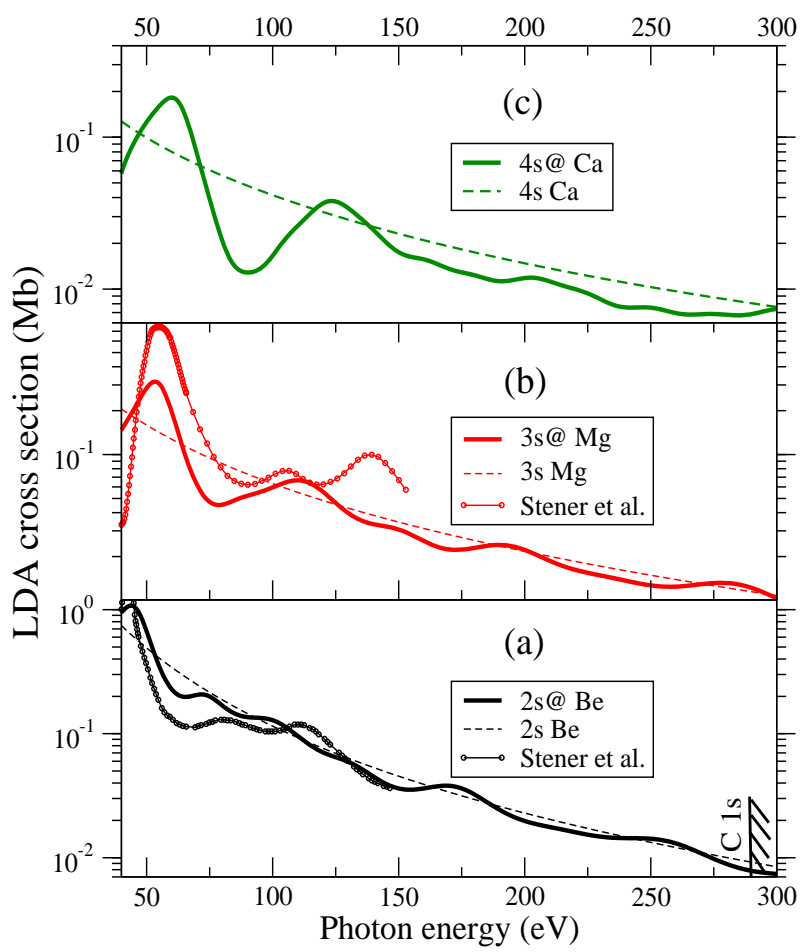

Fig. 3. (Color online) LDA cross sections of the confined and free atomic valence levels for $\mathrm{Be}_{6} \mathrm{C}_{60}$ (a), $\mathrm{MgC}_{60}$ (b) and $\mathrm{Ca} @ \mathrm{C}_{60}$ (c). For the former two systems previous results by Stener et al. [16] are also presented for comparison. The Carbon 1s ionization threshold is noted.

atomic region where the potential changes rapidly. Therefore, since the bound wavefunction $\phi_{\eta \mathrm{s}} @$ are non-vanishing over all these force-sites, as is evident from the probability densities presented in Fig. 1(b), the photoemissions will actually occur from all three sites, significantly interfering with each other owing to the coherence. Additionally, the reflection of the atomic photoelectrons from the hull edges will further enrich the interference, adding features in the modulation that can be best deciphered by the Fourier photo-spectroscopy[19], as we demonstrate below.

The detailed expressions of the general structure of the matrix element has been derived earlier [19,28]. Following Ref. [28], we obtain the $\eta \mathrm{s} @$ ionization amplitude at energies above the $\mathrm{C}_{60}$ plasmon region, as

$$
\begin{aligned}
\mathcal{D}_{\eta \mathrm{s} @} & \sim \mathcal{D}_{\eta \mathrm{s} @}^{\mathrm{atom}}(k) \\
& +A_{\eta \mathrm{s} @}^{\mathrm{refl}}(k)\left[e^{-i k D_{o}} e^{-i V_{0} \frac{2 \Delta}{k}}-e^{-i k D_{i}}\right] \\
& +A_{\eta \mathrm{s} @}^{\mathrm{hull}}(k) e^{-i \frac{V_{0}}{k}}\left[a_{i} e^{-i k R_{i}}-a_{o} e^{-i k R_{o}}\right],
\end{aligned}
$$

in which the photoelectron momentum $k=\sqrt{2\left(E-\epsilon_{\eta \mathrm{s}} @\right)}$ in atomic units, $V_{0}$ is the average depth of the hull potential, and $a_{i}$ and $a_{o}$ are the values of $\eta \mathrm{s} @$ wavefunction at, respectively, $R_{i}$ and $R_{O}$. In Eq. $16, \mathcal{D}_{\eta \mathrm{s} @}^{\text {atom }}$ is very nearly equal to the $\eta$ s matrix element of an isolated atom and the second term on the right hand side denotes the reflection induced oscillations in momentum space with frequencies $D_{i}$ and $D_{o}$, the inner and the outer diameter of the hull; the factor $\exp \left(-i V_{0} \frac{2 \Delta}{k}\right)$ rapidly becomes 


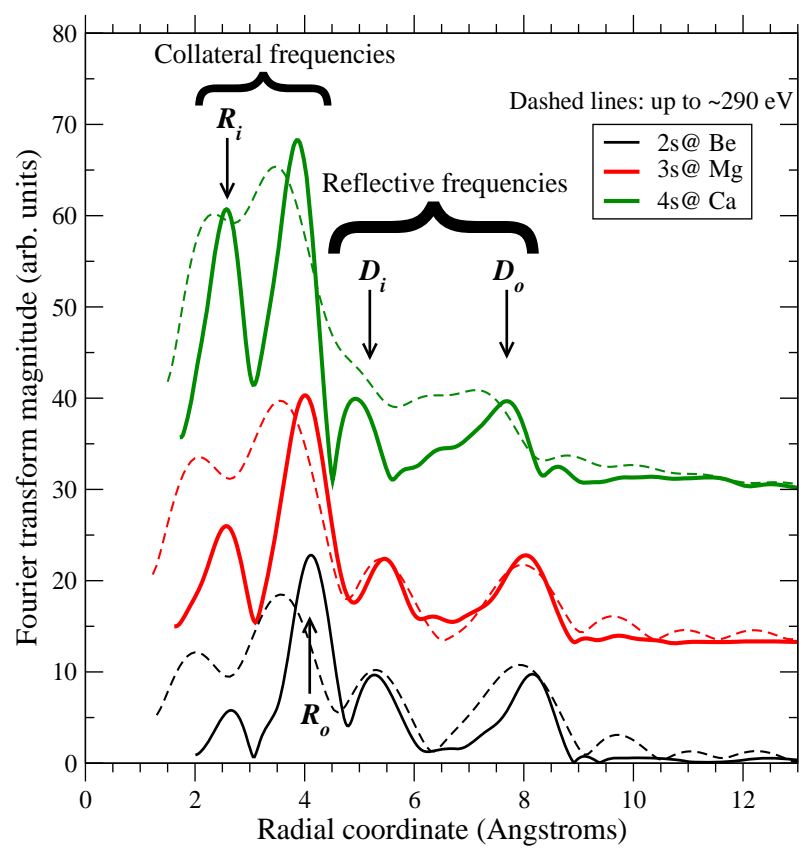

Fig. 4. (Color online) Fourier transform magnitudes of confined-to-free LDA cross section ratios over extended and limited (up to carbon $K$ edge) energy ranges.

unity with increasing $k$. The third term on the right hand side represents the portion of the overlap integral over the hull region, producing two collateral emissions from the hull edges, where non-zero ionizing forces exist [Fig. 1(a)]; as evident, these contributions produce two frequencies, $R_{i}$ and $R_{O}$, in momentum space. Going from confined Be to $\mathrm{Ca}$, since the $\eta \mathrm{s} @$ probability density over the central region slowly diminishes with its corresponding increase in the hull region, the values of $a_{i}$ and $a_{o}$ also grow, as Fig. 1(b) indicates. This evolutionary behavior places, in general, a larger emphasis on the oscillation character of the cross section along the sequence - a feature clearly exhibited in Fig. 3: oscillations are weakest and strongest respectively for $2 \mathrm{~s} @ \mathrm{Be}$ and $4 \mathrm{~s} @ \mathrm{Ca}$.

The determination of the cross section involves interferences among atomic, reflective and collateral hull ionization modes. Upon squaring the modulus of Eq. 16 we find

$$
\sigma_{\eta \mathrm{s} @} \approx \sigma_{\eta \mathrm{s}}^{\text {atom }}+\mathcal{D}_{\eta \mathrm{s} @}^{\mathrm{atom}} \otimes \mathcal{D}_{\eta \mathrm{s} @}^{\mathrm{refl}}+\mathcal{D}_{\eta \mathrm{s} @}^{\text {atom }} \otimes \mathcal{D}_{\eta \mathrm{s} @}^{\text {hull }}
$$

where $\mathcal{D}^{p} \otimes \mathcal{D}^{q}=\mathcal{D}^{p^{*}} \mathcal{D}^{q}+\mathcal{D}^{p} \mathcal{D}^{q^{*}}$, which represents the interferences. Note that since both reflective and collateral effects are generally small relative to the direct ionization, we have disregarded the quadratic reflective, quadratic collateral and reflective-collateral interference terms in Eq. Evidently, the cross section contains the same frequencies as in the matrix element Eq. 16: $D_{i}, D_{o}$ from the reflective and $R_{i}, R_{O}$ from the collateral emissions. This is in clear contrast to the known oscillations in free $\mathrm{C}_{60}$ [18]. In Fig. 4 the Fourier transform magnitudes (FTM) of confined-to-free cross section ratios for $\eta \mathrm{s} @$ ionizations of the three systems are shown. These reciprocal spectra yield four peaks at the expected radial positions. The height of each peak represents the strength of the respective oscillation via the factor in front of the corresponding oscillatory term in Eq. 16. We note the following: (i) The reflective structures are generally smaller than the collateral ones for all the systems. This is because while with increasing photoelectron momentum the reflection probability decreases, a faster electron can better resolve the force structure at the hull edges. (ii) The collateral peaks become progressively taller going from Be to $\mathrm{Ca}$, since $a_{i}$ and $a_{o}$ are increasingly larger along the sequence [Fig. 1(b)]. (iii) In general for all three systems the $R_{O}$ collateral peak is higher then the $R_{i}$ peak, since $a_{o}>a_{i}$ without exceptions.

State-selective measurements of photoelectrons from confined atoms can in principle be carried out by the standard technique of electron spectroscopy. But, the photon energy must not intrude on the carbon $K$-shell continuum to ensure that the $\mathrm{C}^{4+}$ core is not ionized. Hence, we examined if the FTM of cross section ratios for energies not exceeding $290 \mathrm{eV}$ is capable of delineating the effect. Barring some off-sets, the peak positions are very well reproduced (Fig. 3), implying that the effect should be discernible in the experiment and must be included to interpret the data.

\section{Conclusion}

This study of the photoionization of three different alkali earth atoms confined at the center of a $\mathrm{C}_{60}$ molecule reveals a number of insights. The phenomenon of hybridization, the mixing of atomic and shell wave functions, is seen. The details of this hybridization is, however, quite atom-dependent; it depends upon the proximity of atomic energy levels to energy levels of the confining $\mathrm{C}_{60}$ of the same symmetry. In all cases, in the energy vicinity of the giant plasmon in $\mathrm{C}_{60}$, there is a dramatic enhancement of the valence cross section of the caged atom, an effect that is explained in terms of interchannel coupling between the $\mathrm{C}_{60}$ photoionization channels and the atomic channels. The analysis reveals the interference with the atomic ionization amplitude, not the extent of atom- $\mathrm{C}_{60}$ wavefunction mixing, to be the key in determining the relative enhancement that increases from $\mathrm{Mg}$ to $\mathrm{Ca}$ to Be. In addition, well above the plasmonic region, the valence cross sections of all three atoms exhibit confinement resonances, oscillations engendered by the interference of photoelectron waves emitted directly with those (i) scattered from the inner and outer boundary of the confining potential and (ii) emerged directly collaterally from these 7boundaries. These oscillations for each of the atoms differ in detail from one another, but the Fourier transform of the cross sections reveals geometric information on the confining shell which is, of course, exactly the same for all three atoms. However, the orbital-mixing plays a crucial role in altering the intensity of the collateral oscillation component which grows maximally for $\mathrm{Ca}$, since the mixing yields the highest number of collateral electrons for 
this system. Unfortunately, owing to the difficulty of producing these confined atom systems in quantity, there is as yet no experimental scrutiny of these systems. It would be highly desirable to measure these cross sections quantitatively and to look at these systems via photoelectron spectroscopy to separate individual channels. Such studies could answer many questions as well as privde a benchmark for theoretical calculations.

This work is supported by NSF and DOE, Basic Energy Sciences.

\section{References}

1. L. Dunsch and S. Yang, Small 3,(2007) 1298.

2. S.W.J. Scully, E.D. Emmons, M.F. Gharaibeh, R.A. Phaneuf, A.L.D. Kilcoyne, A.S. Schlachter, S. Schippers, A. Müller, H.S. Chakraborty, M.E. Madjet, and J.M. Rost, Phys. Rev. Lett. 94, (2005) 065503.

3. A. Müller, S. Schippers, M. Habibi, D. Esteves, J.C. Wang, R.A. Phaneuf, A.L.D. Kilcoyne, A. Aguilar, and L. Dunsch, Phys. Rev. Lett. 101, (2008) 133001.

4. A.L.D. Kilcoyne, A. Aguilar, A. Müller, S. Cchippers, C. Cisneros, G. Alna'Washi, N.B. Aryal, K.K. Baral, D.A. Esteves, C.M. Thomas, and R.A. Phaneuf, Phys. Rev. Lett. 105, (2010) 213001.

5. V.K. Dolmatov, Advances in Quantum Chemistry. Theory of Confined Quantum Systems, edited by J.R. Sabin and E, Brändas (Academic Press, New York, 2009) Vol. 58, 13ff.

6. M.E. Madjet, T. Renger, D.E. Hopper, M.A. McCune, H.S. Chakraborty, Jan-M Rost, and S.T. Manson, Phys. Rev. A 81, (2010) 013202 and references therein.

7. A.N. Grum-Grzhimailo, E.V. Gryzlova, and S.I. Strakhova, J. Phys. B 44, (2011) 235005.

8. M.Ya. Amusia, L.V. Chernysheva, and V.K. Dolmatov, Phys. Rev. A 84, (2011) 063201.

9. W. Harneit, C. Boehme, S. Schaefer, K. Huebner, K. Fortiropoulos, and K. Lips, Phys. Rev. Lett. 98, (2007) 216601.

10. R.B. Ross, C.M. Cardona, D.M. Guldi, S.G. Sankaranarayanan, M.O. Reese, N. Kopidakis, J. Peet, B. Walker, G.C. Bazan, E.V. Keuren, B.C. Holloway, and M. Drees, Nature Materials 8, (2009) 208.

11. J.B. Melanko, M.E. Pearce, and A.K. Salem, Nanotechnology in Drug Delivery, edited by M.M. de Villiers, P. Aramwit, and G.S. Kwon (Springer, New York, 2009) 105.

12. S. Lo, A.V. Korol and A.V. Solov'yov, Phys. Rev. A 79, (2009) 063201.

13. M. Ya. Amusia, A. S Baltenkov, and L. V. Chernycheva, J. Phys. B 41, (2008) 165201 and references therein.

14. V. K. Dolmatov and S. T. Manson, J. Phys. B 41, (2008) 165001 and references therein.

15. K. Govil, A. J. Siji, and P. C. Deshmukh, J. Phys. B 42, (2009) 065004 and references therein.

16. M. Stener, G. Fronzoni, D. Toffoli, P. Colavita, S. Furlan, and P. Decleva, J. Phys. B 35, (2002) 1421.

17. M.E. Madjet, H.S. Chakraborty, J.M. Rost, and S.T. Manson, J. Phys. B 41, (2008) 105101.

18. A. Rüdel, R. Hentges, H.S. Chakraborty, M.E. Madjet, and J.M. Rost, Phys. Rev. Lett. 89, (2002) 125503.

19. A. Potter, M.A. McCune, R. De, M.E. Madjet, and H.S. Chakraborty, Phys. Rev. A 82, (2010) 033201.
20. A.B. Patel and H.S. Chakraborty, J. Phys. B Fast Track Commun. 44, (2011) 191001.

21. M.E. Madjet, H.S. Chakraborty and S.T. Manson, Phys. Rev. Lett. 99, (2007) 243003.

22. H.S. Chakraborty, M.E. Madjet, J.M. Rost, and S.T. Manson, Phys. Rev. A 78, (2008) 013201.

23. H.S. Chakraborty, M.E. Madjet, T. Renger, Jan-M. Rost, and S.T. Manson, Phys. Rev. A 79, 061201(R) (2009).

24. M.A. McCune, R. De, M.E. Madjet, and H.S. Chakraborty, J. Phys. B Fast Track Commun. 43, (2010) 181001.

25. E. Broclawik and A. Eilmes, J. Chem. Phys. 108, (1998) 3498.

26. M.J. Puska and R.M. Nieminen, Phys. Rev. A 47, (1993) 1181; M.J. Puska and R.M. Nieminen, Phys. Rev. A 49, (1994) 629.

27. O. Gunnerson and B. Lundqvist, Phys. Rev. B 13, (1976) 4274.

28. M.A. McCune, M.E. Madjet, H.S. Chakraborty, Phys. Rev. A 80, (2009) 011201(R). 